\title{
Kernos
}

Revue internationale et pluridisciplinaire de religion grecque antique

$25 \mid 2012$

Varia

\section{Matthew HAYSOM, Jenny WALLENSTEN (éds), Current approaches to religion in ancient Greece}

\section{Vinciane Pirenne-Delforge}

\section{OpenEdition \\ Journals}

\section{Édition électronique}

URL : http://journals.openedition.org/kernos/2049

DOI : 10.4000/kernos.2049

ISSN : 2034-7871

\section{Éditeur}

Centre international d'étude de la religion grecque antique

\section{Édition imprimée}

Date de publication : 26 octobre 2012

Pagination : 332-333

ISSN : 0776-3824

Référence électronique

Vinciane Pirenne-Delforge, « Matthew HAYsom, Jenny wallensten (éds), Current approaches to religion in ancient Greece », Kernos [En ligne], 25 | 2012, mis en ligne le 01 octobre 2012, consulté le 21

septembre 2020. URL : http://journals.openedition.org/kernos/2049 ; DOI : https://doi.org/10.4000/ kernos. 2049

Ce document a été généré automatiquement le 21 septembre 2020.

Kernos 


\title{
Matthew HAYSOM, Jenny WALLENSTEN (éds), Current approaches to religion in ancient Greece
}

\author{
Vinciane Pirenne-Delforge
}

\section{RÉFÉRENCE}

Matthew HAYSOM \& Jenny WALLENSTEN (éds), Current approaches to religion in ancient Greece. Papers presented at a symposium at the Swedish Institute at Athens, 17-19 April 2008, Stockholm, 2011. 1 vol. $16,5 \times 24 \mathrm{~cm}, 315$ p. (Acta Instituti Atheniensis Regni Sueciae, Series in-8 $\left.{ }^{\circ}, 21\right)$. ISBN : 978-91-7916-059-3.

Depuis les années 1980, l'Institut archéologique suédois d'Athènes, sous la houlette de R. Hägg, a livré des actes de colloques d'excellente facture sur le système religieux des Grecs. La grande qualité de ces ouvrages tient à la compétence des intervenants dans les domaines concernés et à la transcription des discussions qui ajoutent très souvent des éléments de réflexion intéressants aux articles eux-mêmes. Le seul bémol est le temps souvent très long qui s'est écoulé entre la tenue de la rencontre et la publication elle-même, introduisant du même coup un déséquilibre entre les articles qui remontent à la période du colloque et ceux que leur auteur a pris la peine de mettre à jour. Le colloque ici recensé s'inscrit explicitement dans la continuité de la tradition de ces rencontres. On n'y trouve plus les discussions, mais le délai de sortie de presse est bien plus court (trois ans au lieu des huit qui se sont écoulés pour le volume Olympian and Chthonian, par exemple1), ce dont on ne peut que féliciter les éditeurs.

2 La moisson de ces Current approaches est incontestablement riche et cette richessemême est liée au foisonnement des intérêts particuliers des contributeurs. Car il s'agit moins de mettre en évidence, comme telles, des approches ou des méthodes actuelles dans l'étude de la religion grecque que de rassembler des travaux de jeunes chercheurs dans le domaine. Le résultat est très intéressant et il s'agit certes d'une forme 
"d'actualité », mais les éditeurs eux-mêmes, dans leur introduction, sont conscients de l'éclatement du propos, en dépit des efforts déployés pour démontrer la pertinence du titre choisi. L'ensemble est donc assez disparate, un peu à la manière d'un volume de varia, qui touche à différents aspects de la religion grecque ${ }^{2}$.

3 L'historiographie ouvre le volume sur une étude de l'interprétation de la figure d'Apollon au XIXe siècle (M. Konaris), Apollon qui se trouve également au centre des trois études suivantes. J. Wallensten s'interroge sur la question de savoir si les liens familiaux mythologiques entre le dieu et sa jumelle Artémis transparaissent dans le corpus de 127 inscriptions dédicatoires qu'elle étudie. L'ancrage délien des documents est si fort dans ce corpus (près de $80 \%$ des dédicaces) qu'il est difficile de répondre à la question initiale de façon pertinente sans élargir le propos à d'autres types de sources (ce dont l'A. est consciente, p. 26). L'Apollon délien a une dimension panhellénique incontestable, ce qui est moins le cas d'un Apollon thessalien, à l'épiclèse de Kerdoos, étudié par M. Mili. Elle analyse finement ce culte peu connu et montre avec pertinence la difficulté de relier le niveau panhellénique et le niveau local dans l'étude des dieux grecs, tout en soulignant la nécessité de poser le problème en ces termes. Quant à l'Apollon de Milet, tant le Delphinios que le Didyméen, A. Herda livre une synthèse en anglais de ses importants travaux, en allemand, sur la procession des Molpes et l'organisation des cultes du dieu dans la cité.

4 Les deux articles suivants posent d'intéressants problèmes de méthode. Le premier (M. Haysom) questionne la continuité entre Crète minoenne et Crète "grecque » en termes religieux, par le biais d'une mise en cause de la notion « d'étrangeté » qui émane souvent de la vision de l'île que l'on trouve dans l'historiographie. Il en ressort une Crète protohistorique plus grecque qu'on ne le lit parfois. Quant à la sempiternelle problématique du couple "privé / public» dans l'étude de la religion grecque, P. Pakkanen la situe d'abord sur un plan herméneutique un peu abstrait pour analyser ensuite ce que l'on peut retirer, en termes rituels, des dépots d'ossements mis au jour dans le sanctuaire de Poséidon à Calaurie.

5 Les thématiques abordées par la suite sont variées: la pompè vue comme escorte (on aurait attendu davantage d'insistance sur l'escorte des animaux de sacrifice) et comme anathèma pour la divinité (A. Kavoulaki) ; les Oschophories analysées en fonction de ce que l'on peut reconstituer de la variété de l'expérience des participants plutôt que selon des modèles interprétatifs " cohérents » et généraux du type "rite de passage " (O. Pilz); l'importance de la dimension musicale des rituels grecs, et plus particulièrement des percussions insuffisamment prises en considération dans les études sur ces questions (K. Kolotourou); l'identification et l'analyse de la vaisselle cultuelle éleusinienne transportée lors de la procession et utilisée lors des rites finaux des mystères (Chr. Mitsopoulou) ${ }^{3}$; l'étude d'un hapax des inventaires déliens en regard d'autres types de source qui permettent d'en éclairer le sens (Cl. Prêtre); l'utilisation de ces mêmes inventaires, à Délos, Athènes et Rhodes, pour inscrire le sanctuaire où ils étaient exposés dans le cadre religieux et politique plus large du monde grec, au-delà de la «polis-religion » (M. Scott).

6 Les deux dernières contributions partagent un même intérêt pour la "vision " en contexte religieux et l'organisation de l'espace, mais l'angle d'attaque est différent. N. Papalexandrou entend souligner l'importance de la vision comme expérience religieuse, notamment la vision d'objets concrets. Il part de questionnements théoriques sur la valeur cognitive des images ou d'autre matériel visuel dans l'antiquité 
et les applique au "big-bang» qu'a dû constituer, d'un point de vue visuel, l'accroissement des artéfacts dans les sanctuaires à partir de la période géométrique, en regard de termes utilisés dans l'épopée (daidalon, thauma idesthai). Étudiant également la disposition des espaces sacrés et les stratégies visuelles en lien avec les statues divines, $\mathrm{J}$. Mylonopoulos rassemble et étudie le corpus des barrières de bois, de métal ou de pierre dont on a trace devant les statues. Il conclut d'une analyse serrée - et d'autant plus méritoire qu'elle est difficile à mener à bien - que les barrières devant les «statues de culte " servaient à marquer une limite physique, symbolique et religieuse entre l'image divine et les fidèles dans les temples qui restaient ouverts de manière plus ou moins régulière. Par conséquent, bon nombre de temples qui ne présentaient pas cette caractéristique architecturale étaient probablement fermés la plupart du temps. Malheureusement, une grande quantité de ces éléments matériels a sans doute irrémédiablement disparu.

\section{NOTES}

1. Voir Kernos 20 (2007), p. 387-392.

2. Voir la table des matières détaillée du volume dans Kernos 24 (2010), p. 363.

3. Cf. Chr. Mitsopoulou, «De nouveaux Kernoi pour Kernos... Réévaluation et mise à jour de la recherche sur les vases de culte éleusiniens », Kernos 23 (2010), p. 145-178. 